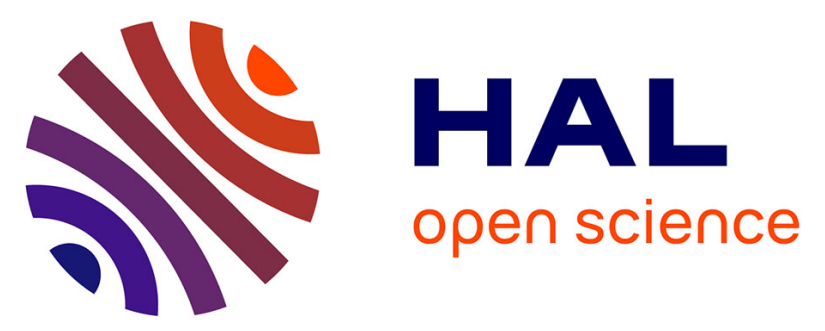

\title{
Understanding and monitoring the capacitance-voltage technique for the characterization of tandem solar cells
}

Cyril Léon, Sylvain Le Gall, Marie-Estelle Gueunier-Farret, Aurore

Brézard-Oudot, Alexandre Jaffré, Nicolas Moron, Laura Vauche, Karim

Medjoubi, Elias Veinberg Vidal, Christophe Longeaud, et al.

\section{To cite this version:}

Cyril Léon, Sylvain Le Gall, Marie-Estelle Gueunier-Farret, Aurore Brézard-Oudot, Alexandre Jaffré, et al.. Understanding and monitoring the capacitance-voltage technique for the characterization of tandem solar cells. Progress in Photovoltaics, 2020, 28 (6), pp.601-608. 10.1002/pip.3235 . hal02439912

\section{HAL Id: hal-02439912 \\ https://hal.science/hal-02439912}

Submitted on 11 Mar 2020

HAL is a multi-disciplinary open access archive for the deposit and dissemination of scientific research documents, whether they are published or not. The documents may come from teaching and research institutions in France or abroad, or from public or private research centers.
L'archive ouverte pluridisciplinaire HAL, est destinée au dépôt et à la diffusion de documents scientifiques de niveau recherche, publiés ou non, émanant des établissements d'enseignement et de recherche français ou étrangers, des laboratoires publics ou privés. 


\section{Understanding and monitoring the capacitance-voltage technique for the characterization of tandem solar cells.}

Cyril Leon ${ }^{1 *}$, Sylvain Le Gall ${ }^{1}$, Marie-Estelle Gueunier-Farret ${ }^{1}$, Aurore Brézard-Oudot ${ }^{1}$, Alexandre Jaffre ${ }^{1}$, Nicolas Moron ${ }^{1}$, Laura Vauche ${ }^{2}$, Karim Medjoubi ${ }^{2}$, Elias Veinberg Vidal ${ }^{2}$, Christophe Longeaud ${ }^{1}$, Jean-Paul Kleider ${ }^{l}$.

${ }^{1}$ Group of electrical engineering - Paris (GeePs), CNRS, CentraleSupélec, Univ. Paris-Sud, Univ. ParisSaclay, Sorbonne Univ., 11 rue Joliot-Curie, 91192 Gif-sur-Yvette, France.

${ }^{2}$ Univ. Grenoble Alpes, CEA, LETI, INES, Grenoble 38000, France.

This paper presents a simple and non-destructive method to determine doping densities and built-in potential of subcells by adapting the well-known capacitance-voltage $(C-V)$ technique to two-terminal (2T) tandem solar cells. Because of the electrical coupling between the two subcells in a monolithic $2 \mathrm{~T}$ tandem solar cell the standard method using a Mott-Schottky plot $\left(1 / C^{2}\right.$ vs $\left.V\right)$ cannot be applied. Using numerical modeling, it is demonstrated that, by under chosen illumination conditions where only one subcell can absorb the light, it is possible to explore the bias dependence of the capacitance and to extract the parameters of the other subcell if the appropriate frequency conditions are present. This method is experimentally applied to an $\mathrm{AlGaAs} / \mathrm{Si}$ tandem cell and parameters of both AlGaAs and Si cells are extracted. Finally, the validity of that method is assessed by the very good agreement obtained when comparing the values extracted from our measurements on the tandem cell to those extracted from measurements on isotype cells and to the values targeted during the fabrication process of the $\mathrm{AlGaAs} / \mathrm{Si}$ tandem solar cell.

Keywords: characterization, tandem, multi-junction, capacitance, electrical coupling, solar cell. 


\section{I INTRODUCTION}

The motivation behind the development and the utilization of tandem solar cells (TSC) lies in the possibility to overcome the Shockley-Queisser limit for single junction solar cell. ${ }^{1}$ For silicon, which dominates the photovoltaic (PV) market, the efficiency of a silicon single-junction solar cell is theoretically limited to $29.4 \% .^{2}$ Coupling silicon with a wide bandgap top cell material in a TSC is one of the approaches to reach higher conversion efficiencies. Actually, III-V semiconductors are among the best top cell partners for silicon based TSCs and an efficiency of $32.8 \%$ has been achieved with a four-terminal mechanically stacked GaAs/Si cell., ${ }^{3,4}$ Regarding the various multi-junction architectures, the two-terminal $(2 \mathrm{~T})$ configuration is the most developed at the industrial level and is easier to implement into PV module technology. For such a monolithic structure, it is of importance to perform characterization at the subcell level in order to identify possible limiting parameters to the whole device performance and find ways for optimization of the tandem cell. However the characterization of each subcell can be challenging and requires to manage with the electrical coupling between top and bottom subcells. ${ }^{5}$ This is particularly true when attempting to measure the capacitance of each subcell, ${ }^{6-8}$ and its dependence on parameters like bias, frequency, temperature, from which different various electronic properties could be extracted. ${ }^{9-12}$ Before performing complex analyses of the capacitance in a TSC, a first step is needed to fully understand how each subcell's contribution to the global capacitance can be separated.

We present in this article a non-destructive method to extract key parameters of the individual subcells in a tandem cell: doping density in the base $\left(N_{\text {base }}\right)$ and barrier height $\left(V_{b}\right)$ of the junction. This method is based on capacitance-voltage $(C-V)$ measurements which is a routine characterization technique for single junctions. In the literature, only a few studies have been published about the use of the $C$ - $V$ technique to characterize multi-junction solar cells at the subcell 
level. In 2017, M. Rutzinger et al. proposed a method based on transient voltage measurement under illumination to indirectly deduce the $C$ - $V$ profile. ${ }^{8}$ This interesting method requires some uncommon tools and could not be easily used by the wider audience. In 2010, C. M. Ruiz et al. used a more simple technique based on $C$ - $V$ measurements under illumination to extract doping densities in the base of each subcell but the mechanisms behind this characterization technique were still misunderstood. ${ }^{6}$ In 2011 , R. Hoheisel et al. used this technique to deduce the subcell photovoltage from a change in the shape of the $C-V$ curve and thus giving details on the distribution of the applied voltage in an illuminated TSC.

In this paper, we first explain in section 2 the capacitance and conductance mechanisms that occur when applying an AC small-signal on a solar cell. From there, we detail the equivalent capacitance of a monolithic TSC under specific illumination. Supported by numerical modeling using Silvaco Atlas software, ${ }^{13}$ we discuss its dependency with the experimental parameters, as the frequency of the AC small-signal or the power of the light bias, in order to define the experimental conditions required to properly access the parameters of the targeted subcell. In section 3 , we experimentally apply this method to an AlGaAs/Si TSC. Using what have been discussed in section 2, we first define the required experimental conditions before measuring the $C-V$ profile of each subcell and extracting the wanted parameters. Moreover, we also discuss the impact of the radiative coupling on our method and how it can be neglected. 


\section{2 |THEORY AND MODELLING}

\section{1 | INTRODUCTION}

There are several contributions to the capacitance in a semiconductor $p-n$ junction. The major ones are the depletion-layer capacitance and the diffusion capacitance. Additional contributions from free carriers in strongly inverted regions or from defects will not be considered here since they play a negligible role for our purpose.

The depletion-layer capacitance, often called also junction capacitance, $C_{J}$ originated from the variation of the width of the depletion zone with the applied voltage. Its expression per unit area in a single $\mathrm{p}-\mathrm{n}$ junction is given by: ${ }^{14}$

$$
C_{J}=\sqrt{\frac{q \varepsilon}{2\left(V_{b}-V-2 k_{B} T / q\right)}\left(\frac{1}{N_{\text {base }}}+\frac{1}{N_{e m i t}}\right)^{-1}}
$$

where $q$ is the elementary charge, $k_{B}$ is the Boltzmann constant, $T$ the temperature, $N_{b a s e}$ and $N_{e m i t}$ are the doping densities in the base and in the emitter, respectively, $\varepsilon$ is the dielectric permittivity of the material, $V_{b}$ is the built-in potential of the junction, $V$ is the applied voltage. In the usual measurement frequency range (up to a few $\mathrm{MHz}$ ), this capacitance is frequency independent since it is related to the response of majority carriers. For uniform doping densities, a Mott-Schottky plot, i.e. a plot of $1 / C^{2}$ versus the reverse applied voltage yields a straight line with a slope related to $N_{\text {base }}$ and $N_{\text {emit }}$ and an intercept voltage, $V_{\text {int }}$, equal to $V_{b}-2 k_{B} T / q$ when extrapolated to $1 / C^{2}=0$. In the case of a highly doped emitter compared to the doping density in the absorber $\left(N_{\text {base }} / N_{\text {emit }}<<\right.$ 1 ), doping density $N_{\text {base }}$ can be deduced from the slope of $1 / C^{2}(V)$ :

$\frac{d\left(1 / C^{2}\right)}{d V}=\frac{-2}{q \varepsilon N_{\text {base }}}$

This can be extended for non-uniform doping to obtain a doping profile, the slope at bias $V$ being related to the doping density at the edge $w$ of the space charge region, calculated from $w=\varepsilon / C$. 
The diffusion capacitance, $C_{D}$, is related to the changes, with the applied bias, of minority carrier densities outside the depletion zone. It is thus proportional to the dark DC current across the junction. The value of $C_{D}$ at low-frequency $\left(C_{D, L F}\right)$ has been derived under specific conditions (base thickness much larger than the minority carrier diffusion length, infinite surface recombination velocity at the contact) by Sze. ${ }^{14}$ He also showed that at high frequency, $C_{D}$ varies as $\omega^{-1 / 2}, \omega$ being the AC small-signal pulsation ( $\omega=2 \pi f$ with $f$ the frequency). We can generalize his approach to the case of solar cells by introducing an effective lifetime, $\tau_{\text {eff }}$, and a generalized expression per unit area:

$$
C_{D}=\frac{C_{D, L F}}{\sqrt{1+\omega \tau_{e f f}}}
$$

with

$$
C_{D, L F}=\frac{q J_{\text {dark }, D C}}{k_{B} T} \times \tau_{e f f}
$$

where $J_{d a r k, D C}$ is the dark DC current density.

Due to their respective bias dependence, $C_{D, L F}$ dominates over $C_{J}$ at large forward bias while $C_{J}$ dominates at reverse and moderate forward bias.

If we now consider a tandem solar cell in dark conditions, the overall device capacitance is given by $1 / C_{T S C}=\sum_{i} 1 / C_{i}$, where $i=1, i=2$ and $i=3$ stand for the top cell, the bottom cell and the tunnel junction, respectively. The capacitance of the tunnel junction $C_{3}$ is much larger than the subcell capacitances due to very high doping densities in the tunneling regions, so its contribution to the total capacitance is generally negligible and will be neglected in the following. Nevertheless, both subcells contribute to the tandem cell capacitance:

$$
\frac{1}{C_{T S C}}=\sum_{i=1,2} \frac{1}{C_{i}}
$$


The weight of each subcell contribution depends on the doping densities and on how the bias applied to the tandem is shared between them. This leads to a non-linear bias dependence of $1 / C_{T S C}{ }^{2}$. In order to illustrate this point, using a TCAD simulator (Silvaco Atlas), ${ }^{13}$ we have performed numerical calculations of the capacitance of a TSC consisting of a top subcell (bandgap energy $\left.E_{g}=1.70 \mathrm{eV}\right)$ and a bottom subcell $\left(E_{g}=1.12 \mathrm{eV}\right.$ representative of silicon). For the top subcell, the doping densities $N_{\text {base }}$ and $N_{\text {emit }}$ were taken equal to $1 \times 10^{16} \mathrm{~cm}^{-3}$ and $1 \times 10^{18} \mathrm{~cm}^{-}$ ${ }^{3}$, respectively, and to $1 \times 10^{15} \mathrm{~cm}^{-3}$ and $1 \times 10^{20} \mathrm{~cm}^{-3}$ for the bottom subcell. Figure $1 \mathrm{~A}$ shows the non-linear behavior of the Mott-Schotky plot for the TSC. In addition we have calculated the capacitance-bias dependence of the top and bottom cells taken separately. As it can be seen in Figure $1 \mathrm{~B}$, the slope of the $1 / C^{2}$ curve of the TSC is bias dependent and its value corresponds to neither of the subcells. This demonstrates that properties of the subcells cannot just be extracted from $C-V$ measurements of the TSC in the dark.
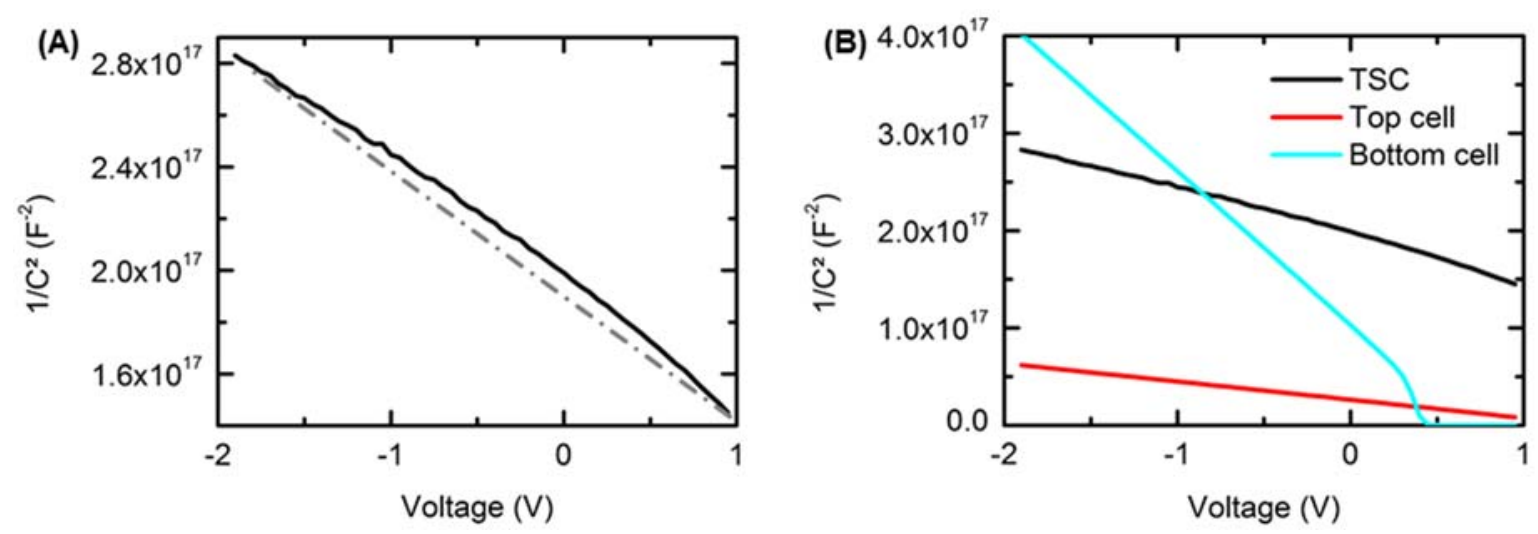

Figure 1 (A) Mott-Schottky plot obtained from numerical calculations for the TSC (black line). The dash-dotted is a straight line to show that the slope of $1 / C^{2}$ is bias dependent. (B) Mott-Schottky plots for the TSC (black line) are compared to those calculated for the subcells alone: top cell (red line) and bottom cell (blue line). Calculations were performed in the dark at $\mathrm{T}=300 \mathrm{~K}$. 


\section{2 | EQUIVALENT CIRCUITS AND LIGHT BIAS}

In order to properly analyze the admittance of the tandem solar cell, one also has to consider additional resistances and conductances. The usual equivalent circuit of a single junction solar cell also involves parasitic series resistance and shunt resistance. These can easily be taken into account, however we will neglect them in our simplified analysis because for high efficiency solar cells they do not play a major role. On the contrary, one has to consider the conductance, $G_{D}$, associated to the diffusion of minority carriers. Its expression per unit area can be generalized from expressions derived by Sze as: ${ }^{14}$

$$
\begin{aligned}
& G_{D}=G_{D, L F} \sqrt{1+\omega \tau_{e f f}} \\
& \text { with } \\
& G_{D, L F}=\frac{q J_{\text {dark }, D C}}{k_{B} T}
\end{aligned}
$$

The schematic equivalent circuit is illustrated in Figure 2A. By using a light bias at a wavelength absorbed by only one of the subcells $\left(\operatorname{TSC}_{\lambda}\right)$, the other subcell remains in the dark and strongly limits the current flowing through the TSC at reverse or moderate forward bias. Let us assume for instance that the top cell is light-biased $\left(\mathrm{TSC}_{\lambda 1}\right)$. If the tandem cell is reverse biased or slightly forward biased (at a bias smaller than the open circuit voltage of the light-biased cell), then the non-absorbing subcell is reverse biased while the top cell operates at forward bias very close to its open-circuit voltage, as illustrated in Figure 2B. At such forward bias, the diffusion conductance cannot be neglected and a competition occurs between $G_{D, 1}$ and $\omega C_{1}, C_{1}$ being the sum of both diffusion and junction top cell capacitances. For the reverse biased bottom cell, the depletion-layer capacitance prevails and its diffusion capacitance and diffusion conductance can be neglected, as shown in Figure 2C. The equivalent tandem solar cell capacitance, $C_{T S C}$, can then be calculated as: 


$$
C_{T S C}=C_{J, 2} \times \frac{1+\left(\frac{C_{1}+C_{J, 2}}{C_{1}}\right)\left(\frac{\omega C_{1}}{G_{D, 1}}\right)^{2}}{1+\left(\frac{C_{1}+C_{J, 2}}{C_{1}}\right)^{2}\left(\frac{\omega C_{1}}{G_{D, 1}}\right)^{2}}
$$
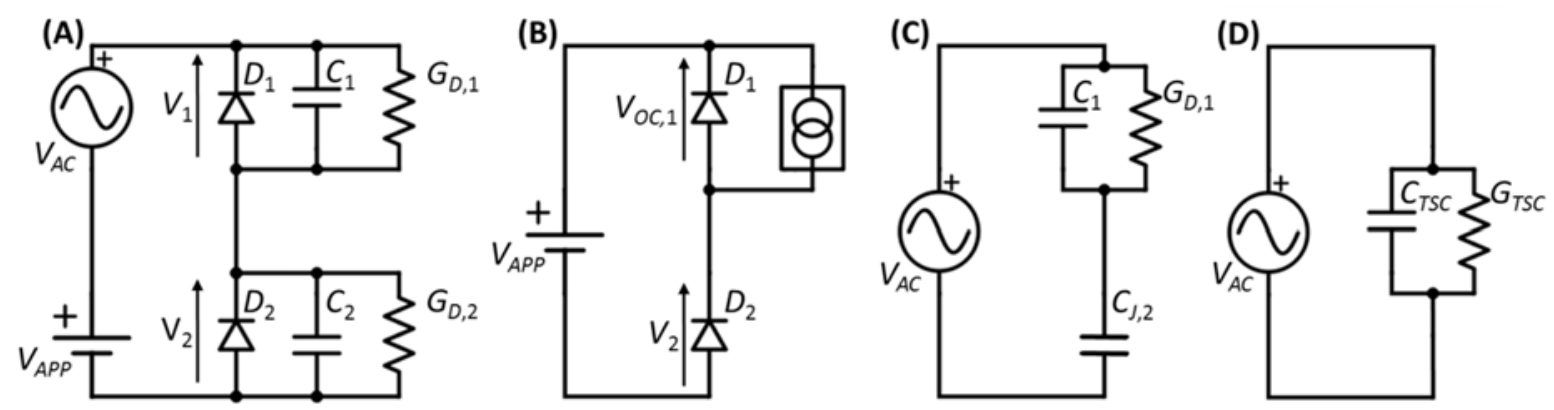

Figure 2 (A) Equivalent circuit of the TSC in dark; (B) DC and (C) AC equivalent circuit of the TSC under reverse bias when the top cell is light-biased; (D) simplified AC equivalent circuit .

In the case where $\omega C_{1} \ll G_{D, 1}$ the equivalent tandem cell capacitance becomes equal to $C_{J, 2}$, and therefore we can probe the bottom subcell because the contribution of the light-biased top subcell to the tandem capacitance becomes insignificant. It is worth mentioning that this will work at low frequency because the diffusion conductance of the light-biased subcell short-circuits its capacitance. If $\omega C_{1} \gg G_{D, 1}$ both subcells contribute to the equivalent tandem capacitance which becomes equal to $\frac{C_{1} C_{J, 2}}{C_{1}+C_{J, 2}}$. Equation 8 and the above argumentation can be adapted to the other case where the bottom cell is light-biased ( $\mathrm{TSC}_{\lambda 2}$ ) simply by swapping the indexed numbers. Nevertheless, the transition between $C_{J, k}$ and $\frac{C_{i} C_{J, k}}{C_{i}+C_{J, k}}$ (where $\mathrm{k}$ is the index of the subcell in the dark) will have a different behavior regarding the kind of material in the TSC. Since in the experimental section we will use an AlGaAs/Si tandem cell as a proof of concept of our method, we have taken the TSC simulated in the section 2.1 with minority carrier lifetimes $\left(\tau_{i}\right)$ equal to $10 \mathrm{~ns}$ and $1 \mathrm{~ms}$ in the top cell and in the bottom cell, respectively. Figure 3 shows the capacitance versus frequency 
curves $(C-f)$ that have been calculated when the TSC is under zero applied DC voltage and under a monochromatic light $\left(\lambda_{1}\right.$ or $\left.\lambda_{2}\right)$ with a photon flux in the range of $1 \times 10^{15} \mathrm{~cm}^{-2} \cdot \mathrm{s}^{-1}$. Additionally, each subcell has been simulated separately in conditions corresponding to those in the TSC: when the TSC is illuminated at the wavelength $\lambda_{1}\left(\lambda_{2}\right)$, calculations are made for the top (bottom) cell alone under the same illumination conditions and at a forward bias close to its opencircuit voltage $V_{O C, 1}\left(V_{O C, 2}\right)$ and for the bottom (top) cell alone in the dark at a reverse bias close to $-V_{O C, 1}\left(-V_{O C, 2}\right)$. Figure $3 \mathrm{~A}$ presents the results obtained under an illumination at $\lambda_{1}$ for the TSC $\left(\operatorname{TSC}_{\lambda 1}\right)$, the bottom cell alone and the serial association of the capacitance of the bottom cell alone and the top cell alone. Figure 3B shows the equivalent calculations under an illumination at $\lambda_{2}$ with the $C$ - $f$ curve of the top cell alone instead of the bottom cell alone. In the conditions of Figure $3 \mathrm{~A}$, the depletion-layer capacitance $C_{J, 2}$ of the bottom cell (lowly doped base) is in the range of 10 $\mathrm{nF} . \mathrm{cm}^{-2}$ while $C_{J, 1}$ of the top cell (higher doped base) is in the range of $100 \mathrm{nF} . \mathrm{cm}^{-2}$. Due to the low lifetime in the top cell, the diffusion capacitance $C_{D, 1}$ and the diffusion conductance $G_{D, 1}$ are frequency independent in the considered frequency range since $\omega \tau_{1} \ll 1$ and their values can be estimated using Equations 4 and 7. The value of $G_{D, 1}$ is linked to the dark DC current at the operating voltage of the cell, which can also be estimated from the short circuit current expected in this cell under light. For AM1.5 illumination, we would expect a value of about $0.8{\mathrm{~S} . \mathrm{cm}^{-2}}^{-2}$ (corresponding to $J_{s c} \approx 20 \mathrm{~mA} \mathrm{~cm}{ }^{-2}$ in a tandem subcell), so for the much weaker monochromatic photon flux of $1 \times 10^{15} \mathrm{~cm}^{-2} \cdot \mathrm{s}^{-1}$ used here, $G_{D, 1}$ is of the order of a few mS.cm ${ }^{-2}$ and $C_{D, 1}=G_{D, 1} \tau_{e f f}$ is thus smaller than $C_{J, 1}$. The short transition in Figure $3 \mathrm{~A}$ thus occurs when $\omega C_{J, 1} \approx G_{D, 1}$, which is in the $\mathrm{kHz}$ range. Also, because $C_{1}$ is significantly larger than $C_{J, 2}$, the two plateaus corresponding to $C_{J, 2}$ and $\frac{C_{1} C_{J, 2}}{C_{1}+C_{J, 2}}$ are close one another. In Figure $3 \mathrm{~B}$, the low frequency diffusion conductance $G_{D, 2}$ is almost the same as $G_{D, 1}$ in Figure $3 \mathrm{~A}$, because this value is determined by the short circuit current of the light absorbing cell. However, due to the much larger effective minority carrier lifetime $(\approx 1 \mathrm{~ms})$ for the bottom cell, the diffusion capacitance $C_{D, 2}$ of the bottom cell is 
much larger than its junction capacitance $C_{J, 2}$ in the low frequency range (LF). At high frequency (HF), $C_{D, 2}$ is frequency dependent and decreases slowly as $\omega^{-1 / 2}$ (Eq. 3 ) so that the total capacitance of the bottom cell saturates at $C_{J, 2}$ at very high frequency, and the tandem capacitance will then saturate at $\frac{C_{J, 2} C_{J, 1}}{C_{J, 2}+C_{J, 1}}$, which is significantly smaller than $C_{J, 1}$. This explains the wider decrease of $C_{T S C}$ in Figure 3B compared to that of Figure 3A, from $C_{J, 1}$ to $\frac{C_{J, 2} C_{J, 1}}{C_{J, 2}+C_{J, 1}}$.
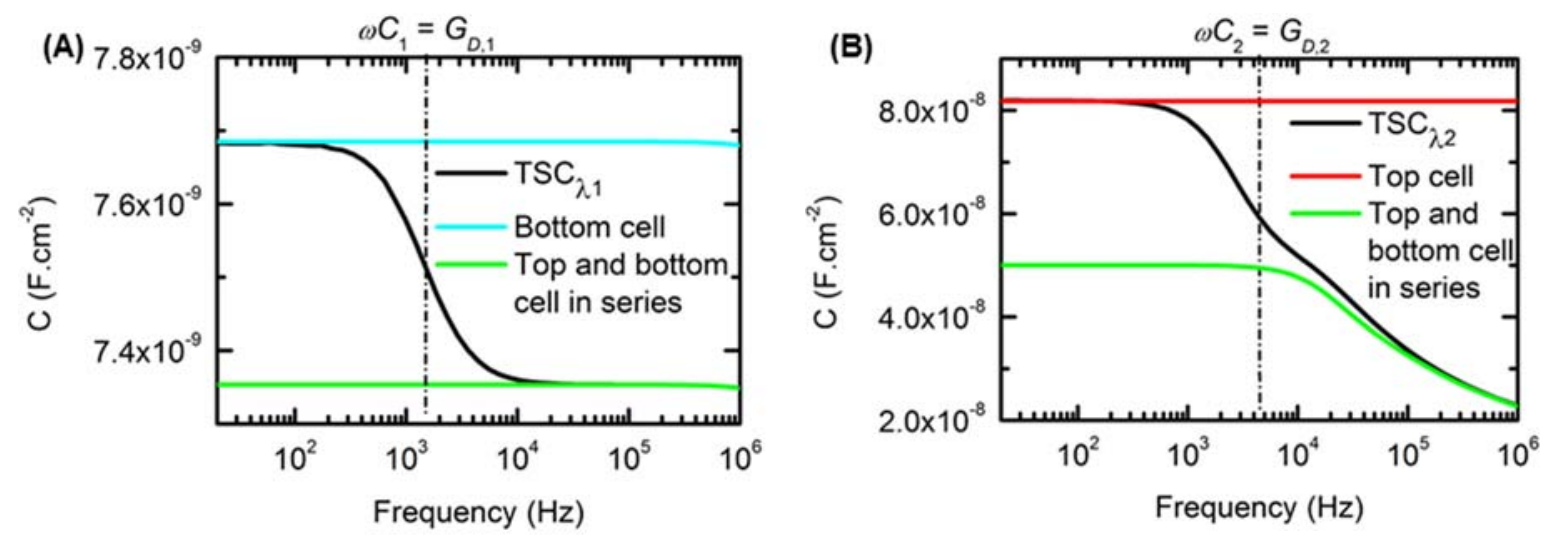

Figure 3 Simulated $C-f$ curve when the TSC is under $0 \mathrm{~V}$ voltage bias and illuminated by a monochromatic light at a wavelength $\lambda_{1}(\mathrm{~A})$ and $\lambda_{2}(\mathrm{~B})$. Calculations have been made for the TSC and for each subcell independently in their operating conditions in the TSC. (A), TSC $\lambda 1$, black line), bottom cell in the dark and polarized at -VOC,1 (blue line), serial association of the bottom cell (dark; -VOC,1) and the top cell (light-biased; $V O C, 1)$ (green line); (B) TSC $\lambda 2$ (black line), top cell in the dark and polarized at - $V_{O C, 2}$ (red line), serial association of the top cell (dark; - $\left.V_{O C, 2}\right)$ (green line) and the bottom cell (light-biased; $\left.V_{O C, 2}\right)$. Dashed dotted lines represent the frequency where $\omega C_{i}=G_{D, i}$.

\section{3 | PHOTON FLUX DEPENDENCY}


All the physical quantities seen above $\left(C_{J}, C_{D}\right.$ and $\left.G_{D}\right)$ are voltage bias dependent and therefore depend on the distribution of the applied voltage between both subcells. By considering the example given in Figure $2 \mathrm{~B}$, the voltage in the bottom cell is $V_{2}=V_{A P P}-V_{O C, 1}$. This means that the photon flux $\left(\Phi_{\lambda i}\right)$ has an influence on the distribution of the voltage in the TSC by changing the value of the open-circuit voltage of the light-biased cell:

$$
V_{O C, i}=\frac{n_{i} k_{B} T}{q} \times \ln \left(\frac{q \times E Q E_{\lambda i} \times \Phi_{\lambda i}}{J_{0, i}}\right)
$$

where $E Q E_{\lambda i}$ is the external quantum efficiency at the considered wavelength $\lambda i, J_{0, i}$ the saturation current in the dark and $n_{i}$ the ideality factor of the light-biased (i-th) subcell. Coupling Equation 9 with Equation 1 and 4 gives:

$$
\begin{aligned}
& C_{J, k}=\sqrt{\frac{q \varepsilon_{k}}{2\left(V_{b, k}-V_{A P P}+\frac{n_{i} k_{B} T}{q} \times \ln \left(\frac{q \times E Q E_{\lambda i} \times \Phi_{\lambda i}}{J_{0, i}}+1\right)-2 k_{B} T / q\right)}\left(\frac{1}{N_{\text {base }, k}}+\frac{1}{N_{e m i t, k}}\right)^{-1}} \\
& C_{D, L F, i}=\frac{q^{2} E Q E_{\lambda i} \times \Phi_{\lambda i}}{k_{B} T} \times \tau_{\text {eff }} \\
& C_{J, i}=\sqrt{\frac{q \varepsilon_{i}}{2\left(V_{b, i}-\frac{n_{i} k_{B} T}{q} \times \ln \left(\frac{q \times E Q E_{\lambda i} \times \Phi_{\lambda i}}{J_{0, i}}+1\right)-2 k_{B} T / q\right)}\left(\frac{1}{N_{\text {base }, i}}+\frac{1}{N_{\text {emit }, i}}\right)^{-1}}
\end{aligned}
$$

The diffusion capacitance $C_{D, i}$ and the diffusion conductance $G_{D, i}$ of the light-biased subcell are linear with the photon flux while the depletion-layer capacitance has a much weaker increase. Considering the subcell in the dark, its depletion-layer capacitance $C_{J, k}$ decreases with the increase of the photon flux absorbed by the light-biased subcell, due to a small negative shift of its operating voltage. Thus, the required condition on the frequency to have $C_{T S C}=C_{J, k}$ (see Fig.3) changes with the photon flux. Considering that the transition observed in Figure 3 occurs at a pulsation $\omega_{c}$ such as $\omega_{c} C_{i} \approx G_{D, i}$ one can write in the low frequency range $\left(\omega_{c} \tau_{e f f}<1\right)$ : 
$\omega_{c}=\frac{C_{D, L F, i}}{C_{D, L F, i}+C_{J, i}} \times \frac{1}{\tau_{e f f, i}}$

which holds if $C_{D, L F, i}<C_{J, i}$. By inserting Equation 11 and Equation 12 in Equation 13, we obtain a quasi-linear dependency of $\omega_{c}$ on $\Phi_{\lambda i}$. This can be seen in Figure 4A, describing the capacitance of the tandem in the frequency - photon flux space $(C-f-\Phi)$ when the top cell is light-biased. Indeed, the white line representing the evolution of $\omega_{c}$ follows a linear trend as predicted.

If $C_{D, L F, i}>C_{J, i}$, which holds if the bottom cell is light-biased owing to the much larger effective lifetime in the bottom cell compared to that in the top cell, we obtain:

$\omega_{c}=\left(\frac{C_{D, L F, i}}{C_{J, i}}\right)^{2 / 3} \times \frac{1}{\tau_{e f f, i}}$

This shown as the white line in Figure 4B.
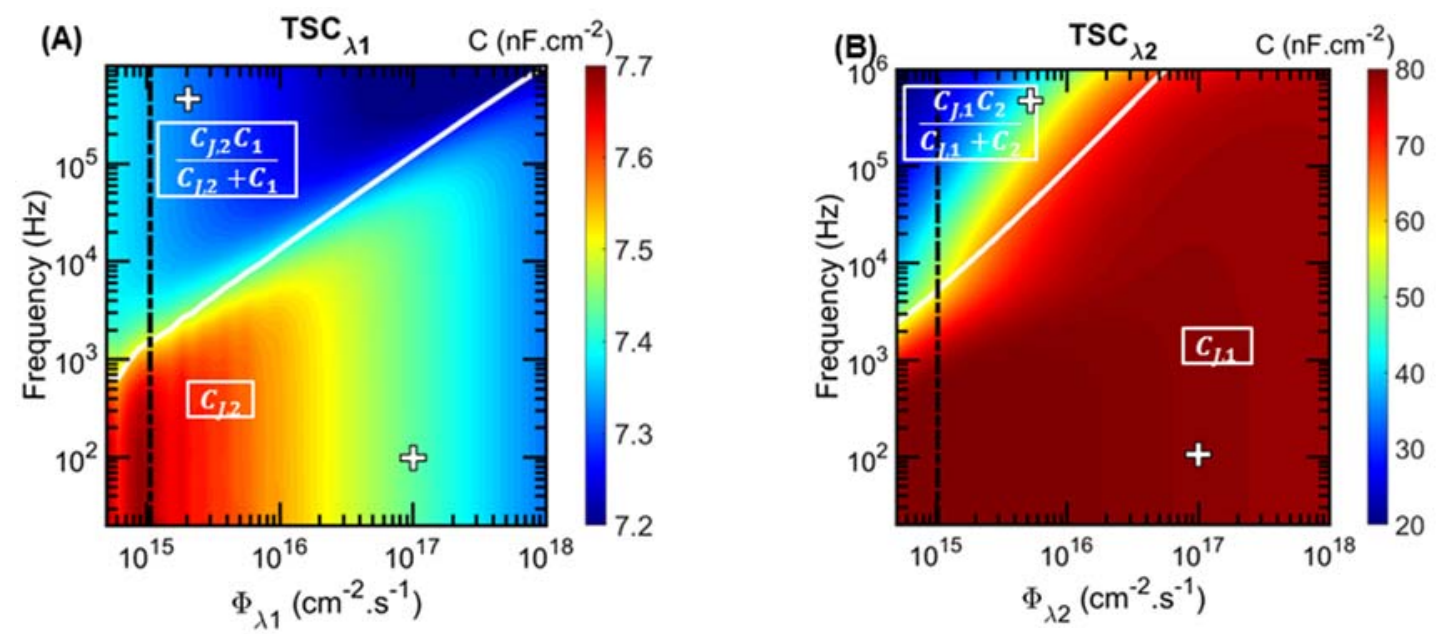

Figure 4 Mapping of the capacitance in the frequency - photon flux space $(C-f-\Phi)$ for (A) the $\mathrm{TSC}_{\lambda 1}$ and (B) the TSC $\lambda 2$. The black dashed lines represent the cutlines from which the curves of the TSC in Figure 3 were obtained. The white lines highlight the evolution of the frequency value for which $\omega C_{i}=G_{D, i}$. The white crosses show the conditions taken for Figure 5. 
Mott-Schottky plots have been simulated for both $\mathrm{TSC}_{\lambda 1}$ and $\mathrm{TSC}_{\lambda 2}$ cases. For each case, two sets of conditions were chosen: a first set of conditions (tagged as "g") taken below the white line in Figure 4 with the frequency of the AC small-signal equal to $100 \mathrm{~Hz}$ and a photon flux equal to $1 \times 10^{17} \mathrm{~cm}^{-2} \cdot \mathrm{s}^{-1}$ and a second set of conditions (tagged as " $\mathrm{b}$ ") taken above the white line with the frequency of the AC small-signal equal to $500 \mathrm{kHz}$ and a photon flux equal to $1 \times 10^{15}$ $\mathrm{cm}^{-2} \cdot \mathrm{s}^{-1}$. Mott-Schottky plots of $\mathrm{TSC}_{\lambda 1}$ and of the bottom cell alone are compared in Figure 5A. Similarly Mott-Schottky plots of $\mathrm{TSC}_{\lambda 2}$ and of the top cell alone are compared in Figure 5B. Both figures illustrate that the influence of the chosen parameters (frequency and photon flux) which is much more pronounced in Figure 5B where the subcell with the lowest doped base is light-biased. . Actually, the relative errors on the extracted quantities (slope of the $1 / C^{2}(V)$ linear part to deduce the doping densities and intercept voltage to deduce built-in potential) for the b-conditions are given in Table 1. The errors are much more important for the $\mathrm{TSC}_{\lambda 2}$ cell which can be explained by considering that the transition between $C_{J, k}$ and $\frac{C_{J, k} C_{i}}{C_{J, k}+C_{i}}$ is larger. This demonstrates that, if considering a TSC with a high difference between the doping densities in the bases, care must be taken to measure the capacitance by fulfilling the required conditions (low frequency and high photon flux). Especially when the subcell with the lowest doped base is light-biased. 

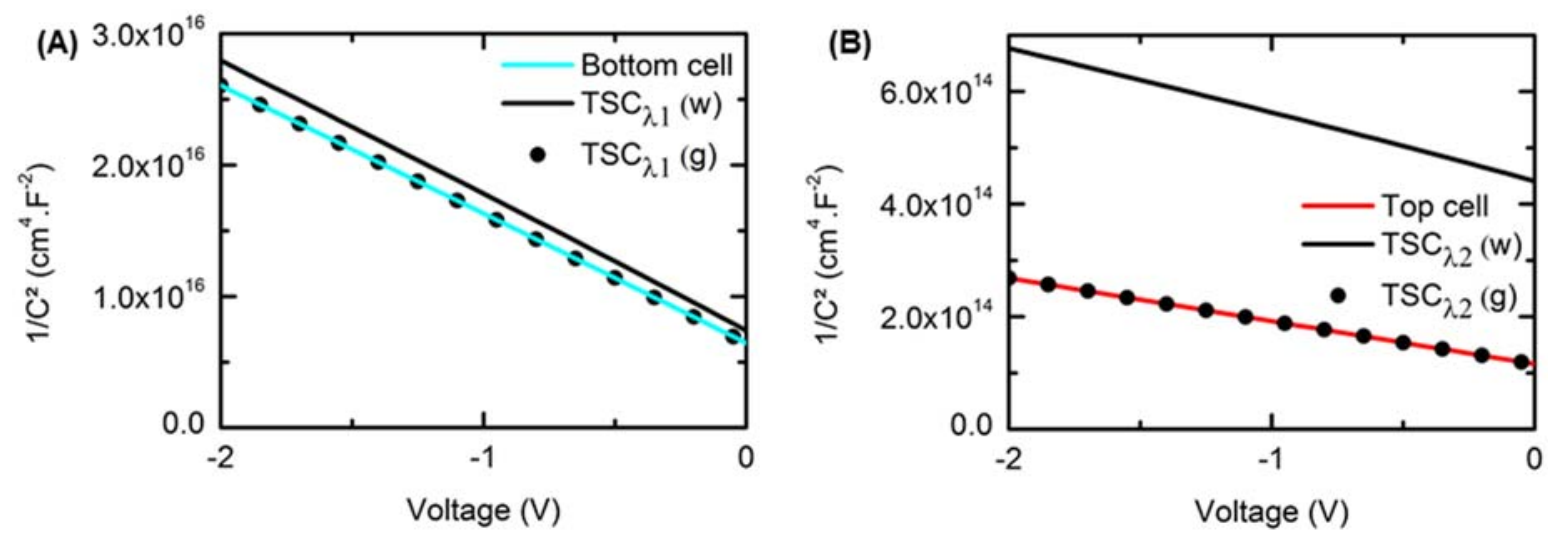

Figure 5 Mott-Schottky plots from numerical calculations of $1 / C^{2}$ values deduced from our simulations of (A) the bottom cell alone in the dark (blue line), the TSC $\lambda 1$ when the required conditions are met (black dots) or not (black line) and (B) the top cell alone in the dark (red line), the $\mathrm{TSC}_{\lambda 2}$ when the required conditions are met (black dots) or not (black line).

Table 1 The relative errors of the extracted parameters from Mott-Schottky plots of the TSC $\lambda 1$ and $\mathrm{TSC}_{\lambda 2}$ simulated with a set of conditions that does not match the requirements.

\begin{tabular}{ccc} 
& $\mathbf{T S C}_{\lambda 1}(\mathbf{w})$ & $\mathbf{T S C}_{\lambda 2}(\mathbf{w})$ \\
Slope & $4.7 \%$ & $54.2 \%$ \\
$V_{\text {int }}$ & $10.7 \%$ & $148.6 \%$ \\
\hline
\end{tabular}




\section{I APPLICATION}

\subsection{REQUIRED CONDITIONS}

In order to experimentally validate our method, we have used an AlGaAs/Si TSC that was fabricated using doping densities in the range of $1 \times 10^{15} \mathrm{~cm}^{-3}$ and $1 \times 10^{17} \mathrm{~cm}^{-3}$ for the base of the Si and AlGaAs cells, respectively, and corresponding emitter doping densities of $1 \times 10^{20}$ $\mathrm{cm}^{-3}$ and $1 \times 10^{18} \mathrm{~cm}^{-3}$ (see Reference [15] for details of the structure and fabrication procedure). The capacitance-voltage measurements were performed using a HP4284A LCR-meter with a AC small-signal of $20 \mathrm{mV}$. The $\mathrm{AlGaAs} / \mathrm{Si}$ tandem cell $\left(0.5 \times 0.5 \mathrm{~cm}^{2}\right)$ was illuminated by monochromatic light coming from a laser either at $\lambda_{1}=405 \mathrm{~nm}$ or at $\lambda_{2}=980 \mathrm{~nm}$. These wavelengths were chosen since according to the EQE ${ }^{15}$ they are predominantly absorbed by one of the subcells. The $405 \mathrm{~nm}$ wavelength is absorbed by the AlGaAs top cell such that the capacitance of the $\mathrm{Si}$ bottom cell can be probed. In the same way, the $980 \mathrm{~nm}$ wavelength is absorbed in the Si bottom cell allowing us to probe the AlGaAs top cell. To fulfill the required conditions described in section 2.3, $C$ - $V$ measurement were performed at $1 \mathrm{kHz}$ with the highest available photon fluxes, i.e. $5.3 \times$ $10^{16} \mathrm{~cm}^{-2} \cdot \mathrm{s}^{-1}$ and $1.3 \times 10^{17} \mathrm{~cm}^{-2} \cdot \mathrm{s}^{-1}$ for the $405 \mathrm{~nm}$ and the $980 \mathrm{~nm}$ light biases, respectively. On this topic, more details can be found in the supplementary materials.

Firstly, we present in Figure 6, the measured $C-f-\Phi$ mapping of the TSC at $0 \mathrm{~V}$ for both wavelengths to find the best experimental conditions. We have limited the frequency range to 500 $\mathrm{Hz}-100 \mathrm{kHz}$ due to the increasing noise below $500 \mathrm{~Hz}$ and to circuit contributions above 100 $\mathrm{kHz}$. The photon flux range is limited by the range of the two light biases from $1 \mathrm{~mW}$ up to 200 $\mathrm{mW}$. However, in order to compare the global shape of the simulated $C-f-\Phi$ mapping with the measured ones, the $\mathrm{X}$ and $\mathrm{Y}$ axes in Figure 6 are kept similar to that in Figure 4. This highlights a good agreement between the simulations of the $C-f-\Phi$ mapping and the measured one. Based on Figure 6 we chose the frequency of the AC small-signal at $1 \mathrm{kHz}$ and the photon flux at $5.3 \times$ 
$10^{16} \mathrm{~cm}^{-2} \cdot \mathrm{s}^{-1}$ and $1.3 \times 10^{17} \mathrm{~cm}^{-2} \cdot \mathrm{s}^{-1}$ for the $405 \mathrm{~nm}$ light bias and the $980 \mathrm{~nm}$ light bias, to extract parameters of the bottom cell and top cell, respectively.
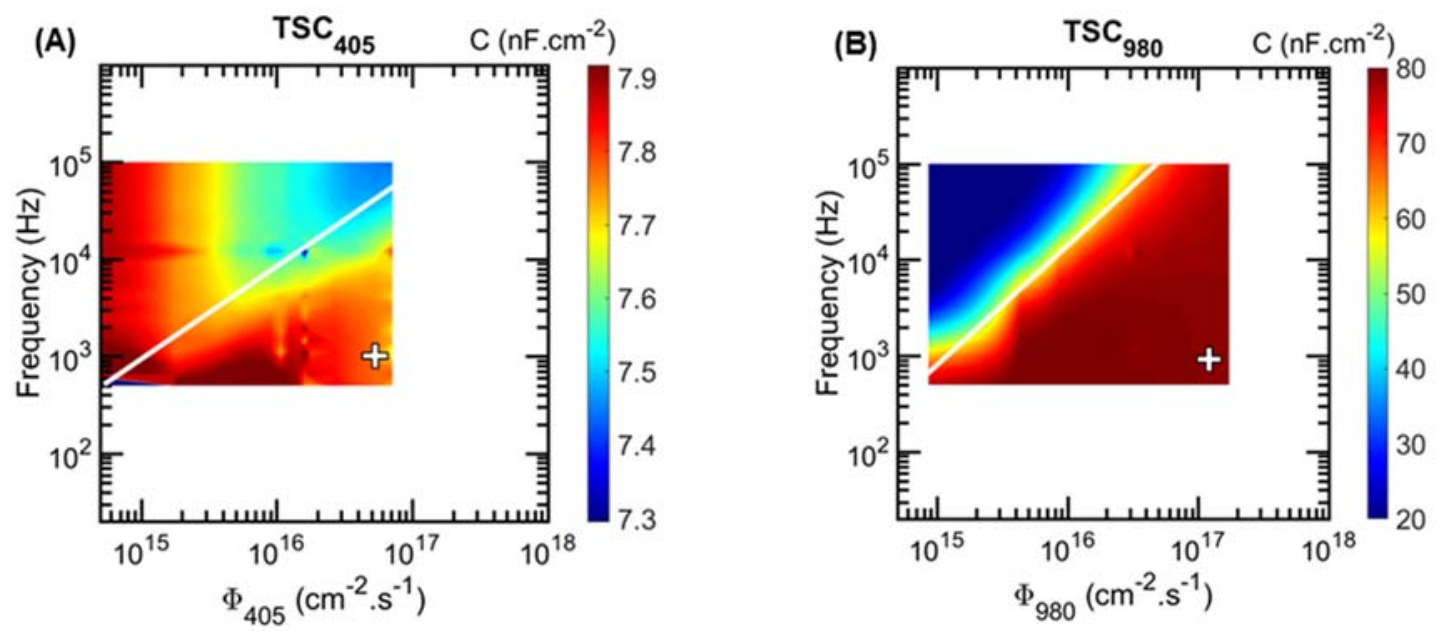

Figure 6 (A) Measured $C-f-\Phi_{405}$ mapping for the $\mathrm{TSC}_{405}$ and (B) Measured $C-f-\Phi_{980}$ mapping for the TSC980. White lines represent the limit where $\omega C_{i} \sim G_{D, i}$. To emphasize the good agreement between measurements and modeling, the axe limits are defined in order to match those of Figure 4. The white crosses show the chosen experimental conditions.

\subsection{Voltage shift and radiative coupling}

As shown in section 2, the voltage bias in the subcell in the dark is $V_{k}=V_{A P P}-V_{O C, i}$. In order to access the $C$ - $V$ curve of the subcell of interest, measurements have to be shifted by the open-circuit voltage of the illuminated cell. The open-circuit voltage is measured between the two terminals of the illuminated TSC when zero current is applied. In so doing, the subcell in the dark will be at $0 \mathrm{~V}$ while the illuminated cell will be at its open-circuit voltage value. However, due to radiative recombinations, it is possible that the illuminated cell emits a small amount of light at an energy equal to its bandgap energy. In a TSC, the other subcell may absorb this emission if its bandgap energy is lower than the energy of the emitted photons from the other cell, which is likely to occur only when the top cell is light-biased. Thus, when measuring $V O C, 1$ when the top subcell 
is light-biased, the bottom cell is not completely in the dark and will contribute to the measured voltage. The voltage shift of the $C-V$ curve will be overestimated and the intercept voltage obtained when extrapolating the Mott-Schottky plot to zero, will then be underestimated. In order to highly decrease the impact of the radiative coupling on the measurement of the open-circuit voltage of the top cell, we first applied a high photon flux $\left(1.3 \times 10^{17} \mathrm{~cm}^{-2} \cdot \mathrm{s}^{-1}\right)$ at $980 \mathrm{~nm}$ absorbed by the Si bottom cell and we measured $V_{O C, 2}$ of the bottom cell (not influenced by radiative coupling). Then we added the TSC light-bias at $405 \mathrm{~nm}$. Because of the logarithmic dependence of $V_{O C}$ on the photon flux, if a small amount of light due to the radiative coupling is added to the high 980 $\mathrm{nm}$ photon flux on the bottom cell, it will not have a significant impact on the bottom cell contribution. The measured voltage can now be assumed to be the sum of $V_{O C, 1}$ and the known $V_{O C, 2}$ and it is possible to determine the top cell contribution alone. In Figure 6A, the evolution of the overestimated $V_{O C, 1}$ and the correct values with the photon flux are presented. Those values are used to shift the Mott-Schottky plots for different photon fluxes and to extract the intercept voltage represented in Figure 6B. Both correct and underestimated $V_{\text {int }}$ values first decrease due to the proximity of the experimental condition with the transition zone where $\omega C_{2} \sim G_{D, 2}$. However the correct $V_{\text {int }}$ value becomes constant which proves that the impact of the radiative coupling has been strongly limited. 
(A)

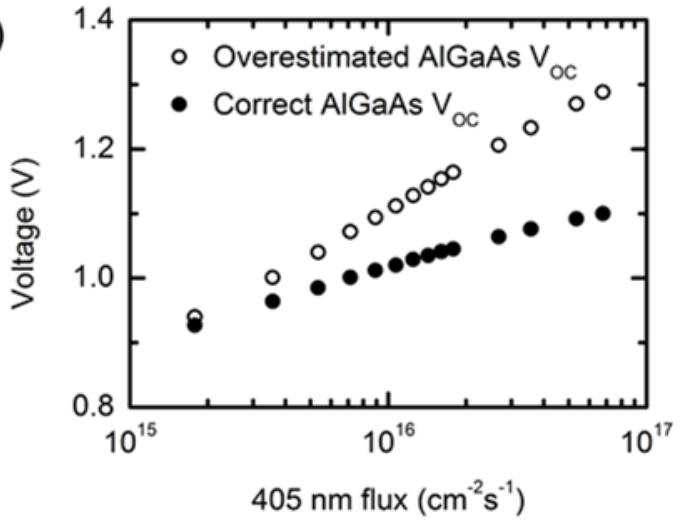

(B)

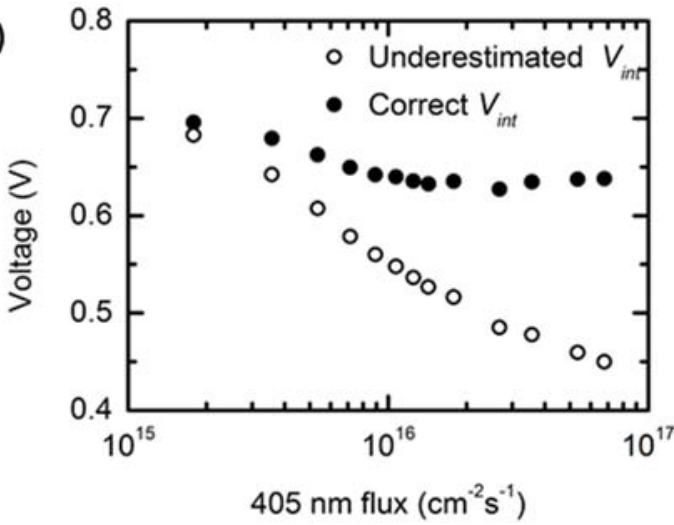

Figure 6 (A) Overestimated $\mathrm{TSC}_{405}$ voltage shift measured when the Si bottom cell is in dark condition (circles) compared with the correct $\mathrm{TSC}_{405}$ voltage shift measured when the $\mathrm{Si}$ bottom cell is strongly illuminated (dots). (B) Comparison between the $V_{\text {int }}$ value of the TSC405 Mott-Schottky plots after voltage correction. Circles represent the underestimated value when shifting with the $V O C$ measured when the Si bottom cell is in dark condition while the dots represent the values when shifting the Mott-Schottky plots with the correct $V_{O C}$ value.

\subsection{PARAMETERS EXTRACTION}

Finally, experimental Mott-Schottky plots of the TSC in the dark and under illumination are compared in Figure 7A and it can be seen that the slopes are very different for each type of illumination and in the dark. In Figure 7B, the Mott-Schottky plot of the capacitance of the TSC405 is compared with measurements performed on a tandem where the top subcell has been shortcircuited resulting in an isotype Si cell. The alignment of both curves after the voltage shift indicates that, using the proposed method, we successfully probed the Si bottom subcell of the TSC. Doping densities in the base of the Si bottom subcell of the TSC and of the isotype Si cell have been determined according to Equation 2 taking account of the relative dielectric permittivity of silicon (11.7). Doping densities in the p-type base and the built-in potentials are summarized in Table 1. Small differences between values can be explained by measurement uncertainties or by slight variations in the fabrication process from one cell to the other. Similarly, the Mott-Schottky 
plot of the TSC980 is shifted by the open-circuit voltage of the bottom subcell in Figure 7C. The doping density in the emitter cannot be neglected and has been considered in Equation 1 when extracting the density in the base with a relative dielectric permittivity value taken equal to $\varepsilon_{s, 1}=12.28$. No isotype AlGaAs cell was available to compare with the TSC980 but the calculated $N_{b a s e, 1}$ value can be compared with the value targeted during the fabrication process which is in the same range. The results summarized in Table 1 allow us to validate the proposed method. Indeed, we obtain a very good agreement between the extracted properties of the subcells of the TSC, those of the isotype Si cell and those targeted during the fabrication process. In addition, it should be emphasized that the method enables to extract doping densities in the base of subcells even if they are very different (more than a factor of 100 in our TSC). 

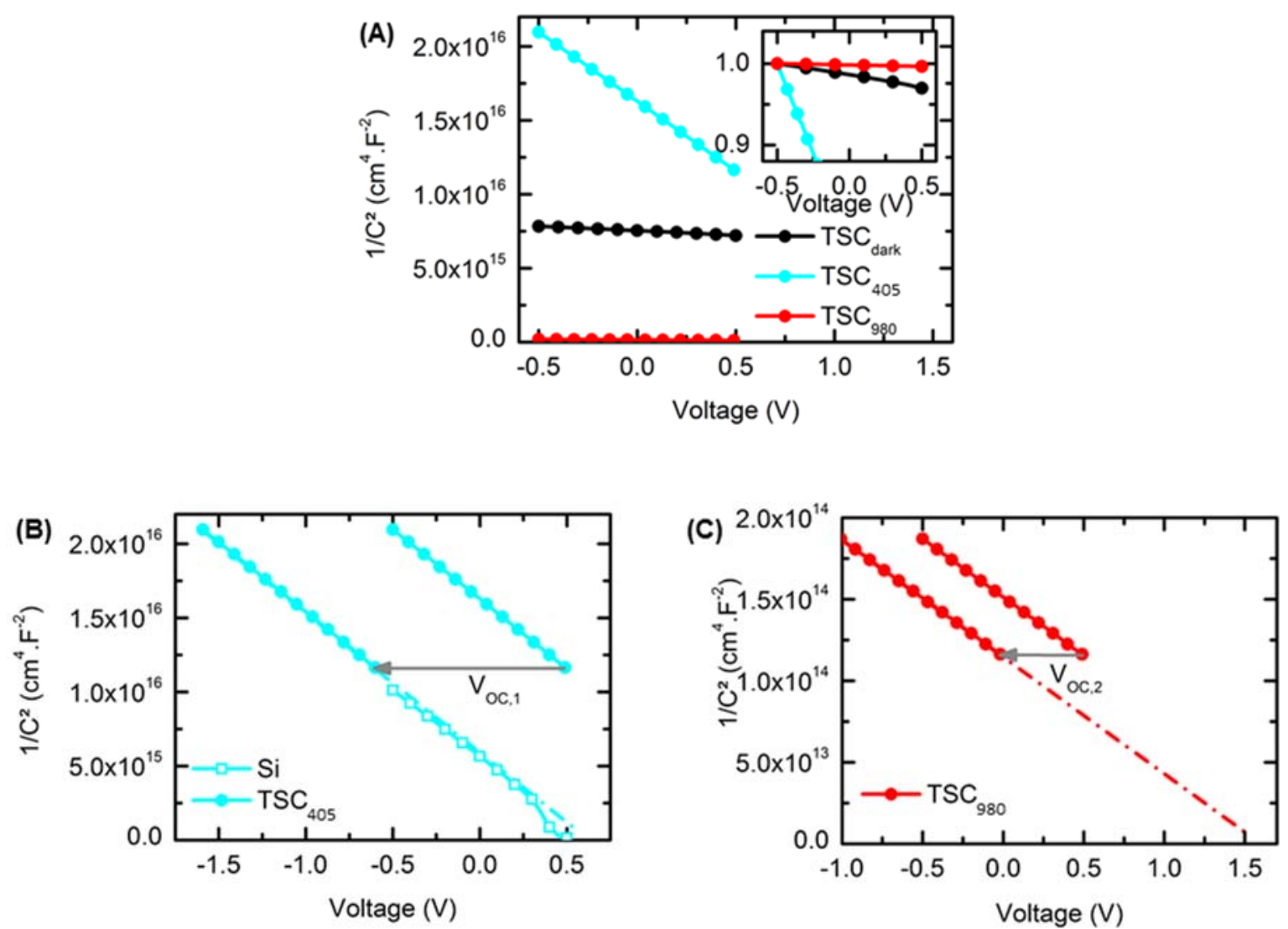

Figure 7 Experimental Mott-Schottky plots: (A) $1 / C^{2}$ curves of the AlGaAs/Si TSC in the dark (black line with dots), under a 405nm (blue line with dots) and a $980 \mathrm{~nm}$ light bias (red line with dots). Inset: normalized $1 / C^{2}$, curves are shifted to the value of the TSC980 at $V=-0.5 \mathrm{~V}$ and normalized to 1 to emphasize that the average slope for the TSC in the dark is different from those under illumination. (B) $1 / C^{2}$ curve of the $\mathrm{TSC}_{405}$ (lines with dots) are shifted by the open-circuit voltage of the top subcell and compared with an isotype Si cell (line with square symbols) to show that the Si bottom subcell of the TSC has been probed. (C) $1 / C^{2}$ curve of the TSC980 (lines with dots) are shifted by the open-circuit voltage of the bottom subcell. 
Table 1 Doping density $\left(N_{b a s e, i}\right)$ in the base, build-in potential $\left(V_{b, i}\right)$ extracted from MottSchottky plots of the TSC405, the TSC980 and the isotype Si cell are compared with targeted doping density values based on the values given in Reference [15] and with expected built-in voltage values based on modeling of targeted the structure.

\begin{tabular}{lll} 
& $N_{\text {base }, i}\left[\mathrm{~cm}^{-3}\right]$ & $V_{b, i}[\mathrm{~V}]$ \\
Targeted for Si & $1 \times 10^{15}$ & 0.69 \\
Measured from Si isotype & $1.3 \times 10^{15}$ & 0.69 \\
Measured from TSC405 & $1.3 \times 10^{15}$ & 0.69 \\
Targeted for AlGaAs & $1 \times 10^{17}$ & 1.59 \\
Measured from TSC980 & $1.7 \times 10^{17}$ & 1.66 \\
\hline
\end{tabular}

\section{I CONCLUSION}

In conclusion, we have presented a method in order to extract key parameters of individual subcells in a tandem solar cell (doping density in the base and potential barrier height) from capacitance measurements performed on the tandem cell under specific light-biasing conditions. Using both analytical and numerical modeling approaches, we have developed a comprehensive analysis of the capacitance response of the tandem solar cell, and detailed the influence of the flux of the light-bias and of the measurement frequency. We have shown that the diffusion conductance and the diffusion capacitance play an essential role, since they allow to screen the contribution of the light-biased cell to the tandem capacitance. Low frequency and high photon flux should be privileged for extracting parameters in a III-V/Si TSC. The method has been applied to an $\mathrm{AlGaAs} / \mathrm{Si}$ TSC. Experimental results are well described by our analysis. The parameters extracted from measurements on the TSC are in good agreement with that obtained from isotype cells, and also with the values targeted in the $\mathrm{AlGaAs} / \mathrm{Si}$ process. The proposed method is simple and nondestructive, and can be extended to other multijunction cells and to other capacitance-based 
characterization techniques such as admittance spectroscopy by integrating the contribution of defects to the analysis.

[1]

Fan JCC, Tsaur B-Y, Palm BJ. In: 16th Photovolt. Spec. Conf. New York: IEEE; 1982. 692-701.

[2]

Richter A, Hermle M, Glunz SW. Reassessment of the limiting efficiency for crystalline silicon solar cells. IEEE J. Photovoltaics 2013. 3(4): 11841191.

[3]

Yu Z, Leilaeioun M, Holman Z. Selecting tandem partners for silicon solar cells. Nat. Energy 2016. 1(11): 16137. Ward JS, Schnabel M, Descoeudres A, Young DL, Woodhouse M, Despeisse M, Ballif C, Tamboli A. Raising the one-sun conversion efficiency of III-V/Si solar cells to $32.8 \%$ for two junctions and $35.9 \%$ for three junctions. Nat. Energy 2017. 2: 17144. 
V,Algora C. Capacitance measurements for subcell characterization in multijunction solar cells. In 35th IEEE Photovolt. Spec. Conf. IEEE; 2010. $708-711$

[7]

Hoheisel R, Schachtner M, Stämmler E, Bett AW. Determination of the subcell photovoltage in multijunction solar cells via voltage-dependent capacitance analysis. Appl. Phys. Lett. 2011.98(25): 251106.

[8]

Rutzinger M, Salzberger M, Gerhard A, Nesswetter H, Lugli P, Zimmermann CG. Measurement of subcell depletion layer capacitances in multijunction solar cells. Appl. Phys. Lett. 2017. 111(18): 183507.

[9]

Hillbrand J, Gold RD. Determination of the impurity distribution in junction diodes from capacitance-voltage measurements. RCA Rev. 1960. 21: $245-252$. Lang DV. Deep-level transient spectroscopy: a new method to characterize traps in semiconductors. J. Appl. Phys. 1974. 45(7): 3023-3032. barriers. J. Appl. Phys. 1975. 46(5): 2204-2214. 
Peaker AR, Markevich VP, Coutinho J. Tutorial: junction spectroscopy techniques and deep-level defects in semiconductors. J. Appl. Phys.2018. 123(16):161559.

Silvaco, Atlas User's Manual, 2016.

Sze SM. Physics of Semiconductor Devices, 2nd edition ed. John Wiley \& Sons, Murray Hill, New Jersey: Wiley-Interscience; 1981.

Vauche L, Veinberg Vidal E, Jany C, Morales C, Decobert J, Dupre C, Mur P. 33rd Eur. Photovolt. Sol. Energy Conf. Exhib. 2017. 1228-1231. 